\title{
COMPUTATIONS OF SIEGEL MODULAR FORMS OF GENUS TWO
}

\author{
NILS-PETER SKORUPPA
}

\begin{abstract}
We explain the basic notions and theorems for doing computations in the theory of Siegel modular forms of degree two, on the full modular group and of even weight. This synopsis concludes with a handy and computationally realistic algorithm for tabulating the Fourier coefficients of such forms and the Euler factors of their Spinor zeta functions. In the second part of this paper we present and discuss some of the results of actual computations which we performed following this algorithm. We point out two (theoretically) striking phenomena that are implied by the results of these computations.
\end{abstract}

\section{INTRODUCTION}

In 1978 Kurokawa computed explicit examples of Siegel modular forms of genus 2 [10]. These examples led to the Saito-Kurokawa conjecture whose proof focussed attention to Jacobi forms, which were then first studied by Eichler and Zagier [5]. Meanwhile, Jacobi forms have been extensively studied and, in the case of genus 1, they are quite well understood. In contrast to this, there are still many gaps in the theory of Siegel modular forms of higher genus, and even in the case of genus 2 many questions are still not answered: Is a Hecke eigenform uniquely determined by its eigenvalues? What is the arithmetic nature of its Fourier coefficients? What is the relation between Hecke eigenforms and Galois representations? Are there Hecke eigenforms of even weight and on the full modular group whose first Fourier-Jacobi coefficient vanishes? What is the relation between the eigenvalues of a Hecke eigenform and the scalar products of its Fourier-Jacobi coefficients (cf. [9])?

At the time of Kurokawa's paper it took much effort and tricky manipulations to produce explicit examples of Siegel modular forms at all. This was mainly due to the lack of computationally realistic formulas for Siegel modular forms. In the past decade there has been much progress in the theory of Jacobi forms, as well as in computer hard- and software development. Exploiting this, one can nowadays rather easily go beyond Kurokawa's computations and produce explicit examples of degree-two forms.

The purpose of this paper is, first of all, to point out how such calculations can be done. Moreover, we actually did such calculations. It turned out that there are two striking, to our knowledge so far unobserved, phenomena, which

Received September 21, 1990.

1991 Mathematics Subject Classification. Primary 11F46, 11F33, 11F30, 11F80; Secondary 65D20, 65-04. 
might deserve further attention. The second purpose of this paper is to describe these phenomena.

We computed the Siegel cusp Hecke eigenforms of genus 2 and even weight on the full Siegel modular group which do not belong to the Maaß-Spezialschar. The first of these forms occurs in weight 20 , and for weight 20 up to weight 32 , which is the range of our computations, the dimensions of the subspaces spanned by such forms is $1,1,2,2,3,4,5$, respectively. Quite expectedly, these Hecke eigenforms can be distinguished by their Hecke eigenvalues (even by the eigenvalue of $T(2)$, the second Hecke operator), and their first FourierJacobi coefficient does not vanish. Let $\Upsilon 20, \Upsilon 22, \Upsilon 24 a, \Upsilon 24 b$, etc. denote these Hecke eigenforms (suitably normalized). Then, in complete analogy to the case of elliptic modular forms, it turned out that for weight $k=28,30,32$ the corresponding Hecke eigenforms are conjugate to each other, i.e., $\Upsilon k b, \Upsilon k c$, etc. are obtained by applying an automorphism of $\mathbb{C}$ to the Fourier coefficients of $\Upsilon k a$.

The first of the phenomena mentioned above is that this does not hold true for the two eigenforms in weight 24 and weight 26 , respectively: these eigenforms have rational Fourier coefficients. This is striking and contradicts common expectation.

The second, though less striking, phenomenon is the existence of congruences modulo various primes (or prime powers) between the Hecke eigenforms $\Upsilon *$ and Hecke eigenforms from the Maaß-Spezialschar. These congruences are trivial in the sense that they can be rather simply verified. On the other hand, they extend to congruences between the corresponding Andrianov (or Spinor) zeta functions and might have some less trivial implications in the (so far nonexisting) theory of Galois representations associated with the Hecke eigenforms $\Upsilon *$.

In the course of the numerical computations we had to handle quite large integers at a reasonable speed (multiplication, factorization) and we needed a certain amount of linear algebra (multiplication of matrices, inversion, characteristic polynomials). All these computations could easily be performed using the software package PARI (cf. [3]). I am very grateful to $\mathrm{H}$. Cohen for introducing me to this system and helping me to take the first steps in using this great piece of software. More extensive tables of the examples considered in this paper will appear in [4].

\section{NotATION}

Throughout, we shall use the following notation:

- $\mathbb{Z}, \mathbb{Q}, \mathbb{C}=$ integers, rational, and complex numbers, $\mathbb{H}=$ Poincaré upper half plane, $\Gamma_{1}=\mathrm{SL}_{2}(\mathbb{Z})=$ elliptic modular group, $\Gamma_{2}=\mathrm{Sp}_{2}(\mathbb{Z})=$ Siegel modular group of genus 2

- $M_{k}\left(\Gamma_{2}\right)=$ space of Siegel modular forms of genus 2 and weight $k$ on the full Siegel modular group $\Gamma_{2}$

- $M_{k}\left(\Gamma_{1}\right)=$ space of elliptic modular forms of weight $k$ on the full modular group $\Gamma_{1}$

- $J_{k, m}=$ space of Jacobi forms on $\mathrm{SL}_{2}(\mathbb{Z})$ of index $m$ and weight $k$

- When the $M$ or $J$ above is replaced by $S$, we always mean the corresponding subspace of cusp forms

- $q=\mathrm{e}^{2 \pi i \tau}, \quad \zeta=\mathrm{e}^{2 \pi i z}, \quad q^{\prime}=\mathrm{e}^{2 \pi i \tau^{\prime}} \quad\left(\tau, \tau^{\prime} \in \mathbb{H}, z \in \mathbb{C}\right)$ 
- Special elliptic modular forms:

$$
\eta=q^{\frac{1}{24}} \prod_{n=1}^{\infty}\left(1-q^{n}\right), \quad \Delta=\eta^{24}, \quad E_{2 k}=1-\frac{4 k}{B_{2 k}} \sum_{l=1}^{\infty} \sigma_{2 k-1}(l) q^{l}
$$

( $B_{k}=k$ th Bernoulli number)

- Special Jacobi forms:

$$
\begin{aligned}
& \phi_{10}=-\Delta \cdot \eta^{-6} \sum_{\substack{r, s \in \mathbb{Z} \\
r \neq s \operatorname{Lod} 2}}(-1)^{r} q^{\left(s^{2}+r^{2}\right) / 4} \zeta^{r}, \\
& \phi_{12}=\Delta \cdot \eta^{-6} \cdot\left(6 \sum_{\substack{r, s \in \mathbb{Z} \\
r \neq s \bmod 2}} s^{2}(-1)^{r} q^{\left(s^{2}+r^{2}\right) / 4} \zeta^{r}-E_{2} \sum_{\substack{r, s \in \mathbb{Z} \\
r \neq s \bmod 2}}(-1)^{r} q^{\left(s^{2}+r^{2}\right) / 4} \zeta^{r}\right)
\end{aligned}
$$

- Special Siegel modular forms of genus two:

$$
\chi_{10}=V\left(\phi_{10}\right), \quad \chi_{12}=V\left(\phi_{12}\right), \quad \chi_{14}=V\left(\phi_{10} E_{4}\right), \quad \chi_{16 a}=V\left(\phi_{10} E_{6}\right) .
$$

The operator $V$ (mapping Jacobi forms to Siegel modular forms) and the fact that $\phi_{10}$ and $\phi_{12}$ are elements of $S_{10,1}$ and $S_{12,1}$ will be explained below (cf. the second theorem and the proposition in $\S 1$ ). For a basic reference on Siegel modular forms, we refer to [6]; for Jacobi forms, cf. [5].

\section{TheOREMS FOR COMPUTING SIEgel MODUlaR FORMS}

We are interested in Siegel modular forms of even integral weight on the full modular group. Any such form $F$ has a Fourier expansion of the form

$$
F=\sum_{\substack{r, n, m \in \mathbf{Z} \\ r^{2}-4 m \leq 0 \\ n, m \geq 0}} a_{F}(n, r, m) q^{n} \zeta^{r} q^{\prime m}
$$

in which only those Fourier coefficients $a_{F}(n, r, m)$ are possibly nonzero where the binary quadratic form $[n, r, m]$ (i.e., the form $n X^{2}+r X Y+m Y^{2}$ ) is positive semidefinite. Moreover, the Fourier coefficient $a_{F}(n, r, m)$ depends only on the $\mathrm{GL}_{2}(\mathbb{Z})$-equivalence class of the binary quadratic form $[n, r, m]$. Thus, one wants to compute the Fourier coefficients $a_{F}(Q)$ for all positive semidefinite $\mathrm{GL}_{2}(\mathbb{Z})$-reduced quadratic forms $Q$. The essential ingredient to tabulate these Fourier coefficients is the following theorem of Igusa, which describes the structure of the graded ring of all Siegel modular forms of even weight on $\mathrm{Sp}_{2}(\mathbb{Z})$.

Theorem [7]. Let $\psi_{4}, \psi_{6}, \chi_{10}, \chi_{12}$ be nonzero forms in the one-dimensional spaces $M_{4}\left(\Gamma_{2}\right), M_{6}\left(\Gamma_{2}\right), S_{10}\left(\Gamma_{2}\right), S_{12}\left(\Gamma_{2}\right)$, respectively. Then

$$
M_{2 *}\left(\Gamma_{2}\right):=\bigoplus_{k \in \mathbb{Z}} M_{2 k}\left(\Gamma_{2}\right)=\mathbb{C}\left[\psi_{4}, \psi_{6}, \chi_{10}, \chi_{12}\right],
$$

i.e., the modular forms $\psi_{4}, \psi_{6}, \chi_{10}, \chi_{12}$ are algebraically independent and any element of $M_{2 *}\left(\Gamma_{2}\right)$ can be written as a polynomial in these functions.

According to Igusa's theorem we have to look for a good method to compute the $\psi_{4}, \ldots$. The most convenient method is provided by the following theorem, 
which is essentially due to Maaß. Recall that any element $\phi$ of $J_{k, 1}$ has a Fourier expansion of the form

$$
\phi=\sum_{\substack{D, r \in \mathbf{Z}, D \leq 0 \\ D \equiv r^{2} \bmod 4}} C_{\phi}(D) q^{\left(r^{2}-D\right) / 4} \zeta^{r}
$$

(cf. [5, Theorem 2.2]).

Theorem [11]. For any integer $k \geq 0$, the map

$$
\begin{gathered}
\phi=\sum_{\substack{D, r \in \mathbf{Z}, D \leq 0 \\
D \equiv r^{2} \bmod 4}} C_{\phi}(D) q^{\left(r^{2}-D\right) / 4} \zeta^{r} \mapsto \sum_{\substack{n, r, m \in \mathbf{Z} \\
r^{2}-4 m n \leq 0 \\
n, m \geq 0}} a(n, r, m) q^{n} \zeta^{r} q^{\prime m}, \\
a(n, r, m):=\sum_{a \mid(n, r, m)} a^{k-1} C_{\phi}\left(\frac{r^{2}-4 m n}{a^{2}}\right), \quad a(0,0,0):=-\frac{B_{2 k}}{4 k} \cdot C_{\phi}(0),
\end{gathered}
$$

defines a Hecke equivariant embedding

$$
V: J_{k, 1} \hookrightarrow M_{k}\left(\Gamma_{2}\right) .
$$

It maps cusp forms to cusp forms, and Eisenstein series to Eisenstein series.

The Siegel modular forms occurring in the image of $V$ are called MaaßSpezialformen. To compute such forms, we need to compute Jacobi forms of index 1. Via the following proposition, this is reduced to the computation of elliptic modular forms on the full modular group.

Proposition [13]. Let

$$
\begin{aligned}
A & =\eta^{-6} \sum_{\substack{r, s \in \mathbf{Z} \\
r \neq s \bmod 2}} s^{2}(-1)^{r} q^{\left(s^{2}+r^{2}\right) / 4} \zeta^{r} \\
& =2+q\left(2 \zeta^{2}-8 \zeta+12-8 \zeta^{-1}+2 \zeta^{-2}\right)+\cdots, \\
B & =\eta^{-6} \sum_{\substack{r, s \in \mathbf{Z} \\
r \neq s \bmod 2}}(-1)^{r} q^{\left(s^{2}+r^{2}\right) / 4} \zeta^{r} \\
& =2-\zeta-\zeta^{-1}+q\left(2 \zeta^{2}-8 \zeta+12-8 \zeta^{-1}+2 \zeta^{-2}\right)+\cdots
\end{aligned}
$$

Then, for any integer $k$, the map

$$
(f, g) \mapsto \frac{k}{2} f A-\left(q \frac{d}{d q} f\right) B+g B
$$

defines an isomorphism

$$
I: M_{k}\left(\Gamma_{1}\right) \oplus S_{k+2}\left(\Gamma_{1}\right) \stackrel{\approx}{\rightarrow} J_{k, 1} .
$$

Proof. For the convenience of the reader, we sketch the short proof of this theorem. Set

$$
\vartheta_{\rho}=\sum_{\substack{r \in Z \\ r \equiv \rho \bmod 2}} q^{r^{2} / 4} \zeta^{r} \quad(\rho=0,1) .
$$

In terms of these fundamental theta functions, any $\phi \in J_{k, 1}$ can be written as

$$
\phi=h_{0} \vartheta_{0}+h_{1} \vartheta_{1}, \quad h_{\rho}=\sum_{\substack{D \leq 0, D \equiv \rho \bmod 2}} C_{\phi}(D) q^{-\frac{D}{4}} \quad(\rho=0,1) .
$$


From this equation we obtain

$$
\left(h_{0}, h_{1}\right) W=\left(\phi(\tau, 0), \phi_{z z}(\tau, 0)\right), \quad W=\left(\begin{array}{ll}
\vartheta_{0}(\tau, 0) & \vartheta_{0, z z}(\tau, 0) \\
\vartheta_{1}(\tau, 0) & \vartheta_{1, z z}(\tau, 0)
\end{array}\right),
$$

where the subscript $z z$ indicates second partial derivative with respect to $z$. But $W$ is invertible: namely, using $\operatorname{det}(W)=2(2 \pi i)^{2} q^{\frac{1}{4}}+\mathscr{O}\left(q^{\frac{5}{4}}\right)$, and the wellknown transformation laws satisfied by the $\vartheta_{\rho}$ (cf. $[5, \S 5]$ ), one easily verifies that $\operatorname{det}(W)^{4}$ is an element of $S_{12}\left(\Gamma_{1}\right)$, i.e., equals $\Delta$ up to multiplication by a scalar; whence

$$
\operatorname{det}(W)=2(2 \pi i)^{2} \eta^{6}
$$

Using this to write down the inverse matrix of $W$, we find

$$
W^{-1}\left(\begin{array}{l}
\vartheta_{0} \\
\vartheta_{1}
\end{array}\right)=\left(\begin{array}{c}
A / 2 \\
-B / 2(2 \pi i)^{2}
\end{array}\right)
$$

Summing up, we finally have

$$
\phi(\tau, z)=\frac{1}{2} \phi(\tau, 0) A-\frac{1}{2(2 \pi i)^{2}} \phi_{z z}(\tau, 0) B,
$$

which can also be written as

$$
\phi=\frac{k}{2} f A-\left(q \frac{d}{d q} f\right) B+g B,
$$

where

$$
f=\frac{\phi}{k}, \quad g=\frac{1}{2 \pi i k} \phi_{\tau}(\tau, 0)-\frac{1}{2(2 \pi i)^{2}} \phi_{z z}(\tau, 0) .
$$

From the transformation laws of $\phi$ under $\Gamma_{1}$ it is easily deduced that $f$ and $g$ are elliptic modular forms on the full modular groups of weight $k$ and $k+2$, respectively, and $g$ is even a cusp form. Vice versa, it can be shown, using the transformation laws for the $\vartheta_{\rho}$, that the right side of the last equation for $\phi$ always defines an element of $J_{k, 1}$ if $f \in M_{k}\left(\Gamma_{1}\right)$ and $g \in S_{k+2}\left(\Gamma_{1}\right)$. This completes the proof of the proposition.

Note that $\phi$ is a cusp form if and only if $f$ is a cusp form. Hence, the first Jacobi cusp forms of index 1 occur in weights 10 and 12; these are the two special Jacobi forms listed in Notation. In fact, one has

$$
\phi_{10}=I(0,-\Delta), \quad \phi_{12}=I(\Delta, 0) .
$$

Note that these Jacobi forms have integral Fourier coefficients and that they are normalized in the sense $C_{\phi_{10}}(-3)=C_{\phi_{12}}(-3)=1$.

Moreover, the proposition and its supplement concerning cusp forms shows that $\operatorname{dim} S_{k, 1}=\operatorname{dim} S_{k}\left(\Gamma_{1}\right)+\operatorname{dim} S_{k+2}\left(\Gamma_{1}\right)=\operatorname{dim} M_{k-12}\left(\Gamma_{1}\right)+\operatorname{dim} M_{k-10}\left(\Gamma_{1}\right)$. Since $\phi_{10}$ and $\phi_{12}$ are obviously linearly independent over the ring

$$
M_{*}\left(\Gamma_{1}\right):=\bigoplus_{k \in \mathbb{Z}} M_{k}\left(\Gamma_{1}\right)=\mathbb{C}\left[E_{4}, E_{6}\right]
$$

$\left(\phi_{12} / \phi_{10}\right.$ does depend on $z$ ), we conclude

$$
S_{k, 1}=M_{k-10}\left(\Gamma_{1}\right) \phi_{10} \oplus M_{k-12}\left(\Gamma_{1}\right) \phi_{12} .
$$


To sum up, by the proposition, or the last equation, we have explicit formulas for Jacobi forms of index 1. Via Maaß's theorem we then also have explicit formulas for the generators of the ring $M_{2 *}\left(\Gamma_{2}\right)$, namely,

$$
\psi_{4}=V\left(I\left(E_{4}, 0\right)\right), \quad \psi_{6}=V\left(I\left(E_{6}, 0\right)\right), \quad \chi_{10}=V\left(\phi_{10}\right), \quad \chi_{12}=V\left(\phi_{12}\right) ;
$$

hence, we have such explicit formulas for any Siegel modular form. These formulas are easily implemented on a computer to tabulate the Fourier coefficients of a basis of Siegel modular forms of given weight $k$. The only parts of this procedure which are computationally expensive are the multiplications of Siegel modular forms. To avoid some of these multiplications, it is reasonable to generate at least the Maaß-Spezialschar of a given weight directly, i.e., by applying Maaß's theorem and the above proposition directly instead of writing members of the Spezialschar as polynomials in $\psi_{4}$ to $\chi_{12}$. We followed this procedure for our numerical calculations (cf. $\S 4$ ).

\section{HECKE THEORY}

In this section we recall the theorems concerning the Hecke theory of genus 2 forms, which are necessary to handle and to compute Hecke eigenforms.

Theorem [1, p. 228, Example 4.2.10]. Let $k, l$ be integers, and $l \geq 1$; let

$$
F=\sum_{Q=[n, r, m] \geq 0} a(Q) q^{n} \zeta^{r} q^{\prime m}, \quad T(l) F=\sum_{Q=[n, r, m] \geq 0} a^{*}(Q) q^{n} \zeta^{r} q^{\prime m},
$$

where $F$ is an element of $M_{k}\left(\Gamma_{2}\right)$ and $T(l)$ denotes the lth Hecke operator on this space. Then

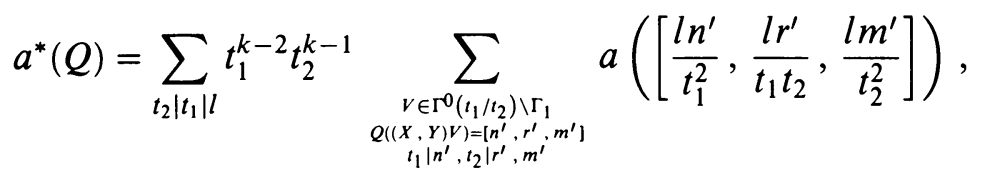

where the inner sum is over a complete set of representatives $V$ for $\Gamma^{0}\left(\frac{t_{1}}{t_{2}}\right) \backslash \Gamma_{1}$ satisfying the stated conditions, and where $\Gamma^{0}(N):=\left(\begin{array}{cc}\mathbb{Z} & N \mathbb{Z} \\ \mathbb{Z} & \mathbb{Z}\end{array}\right) \cap \Gamma_{1}$.

We mention some special cases of the above theorem, which are important for our numerical computations:

To begin with, assume that we have computed sufficiently many coefficients of a basis of a Hecke invariant subspace of Siegel modular forms on $\Gamma_{2}$, and that we want to compute the Hecke eigenforms. The obvious method is to compute the matrix of $T(p)$ for some small prime number $p$ and to diagonalize it. Thus, one needs in particular the formula for the action of $T(p)$ on the Fourier coefficients $a(Q)$ of a given form $F$. By the above theorem it is easily verified that such an explicit formula can be given as follows:

$$
\begin{aligned}
a_{T(p) F}(n, r, m)= & a(p[n, r, m])+p^{2 k-3} a\left(\frac{p}{t^{2}}[n, r, m]\right)+p^{k-2} a\left(\frac{m}{p}, r, p n\right) \\
& +p^{k-2} \sum_{\nu \bmod p} a\left(\frac{n+r \nu+m \nu^{2}}{p}, r+2 m \nu, p m\right) .
\end{aligned}
$$

Here, $p$ is any prime number, and we set $a(Q)=0$ if $Q$ is not integral. 
Secondly, assume that we have computed sufficiently many Fourier coefficients $a(Q)$ of a Hecke eigenform $F$, and that we want to compute the $p$ th Euler factor of the Andrianov zeta function of $F$. As explained in the next theorem, we thus need to compute the eigenvalues $\lambda_{p}$ and $\lambda_{p^{2}}$ of $T(p)$ and $T\left(p^{2}\right)$, respectively. From the above theorem we find the formulas

$$
\begin{aligned}
\lambda_{p} a(1,1,1)= & a(p, p, p)+p^{k-2}\left(1+\left(\frac{p}{3}\right)\right) a(1,1,1), \\
\lambda_{p} a(p, p, p)= & a\left(p^{2}, p^{2}, p^{2}\right)+p^{2 k-3} a(1,1,1)+p^{k-2} a\left(1, p, p^{2}\right) \\
& +p^{k-2} \sum_{\nu \bmod p} a\left(1+\nu+\nu^{2}, p(1+2 \nu), p^{2}\right), \\
\lambda_{p^{2}} a(1,1,1)= & a\left(p^{2}, p^{2}, p^{2}\right)+p^{k-2} \sum_{\substack{\nu \bmod p \\
1+\nu+\nu^{2}=0 \bmod p}} a\left(1+\nu+\nu^{2}, p(1+2 \nu), p^{2}\right) \\
& +p^{2 k-4}\left(\left(\frac{p}{3}\right)+\left(\frac{p^{2}}{3}\right)\right) a(1,1,1) .
\end{aligned}
$$

The eigenvalue $\lambda_{p}$ can be computed from the first of these equations (if $a(1,1,1) \neq 0)$. However, it is computationally expensive to compute $\lambda_{p^{2}}$ directly from the third equation, since one would need to compute $a\left(p^{2}, p^{2}, p^{2}\right)$, i.e., one would need to compute a Fourier coefficient $a(Q)$ where the discriminant of $Q$ is of order $p^{4}$. To avoid this, one should eliminate the $a\left(p^{2}, p^{2}, p^{2}\right)$ in the third formula, using the second one. One can go even one step further and eliminate then the $a(p, p, p)$, using the first formula, so as to obtain a formula expressing $\lambda_{p^{2}}$ in terms of $a(1,1,1)$ and $a(Q)$ with $Q$ primitive and of discriminant $-3 p^{2}$. The precise formula that one obtains in this way is

$$
\begin{aligned}
\lambda_{p^{2}} a & (1,1,1) \\
= & {\left[\lambda_{p}^{2}-\lambda_{p} p^{k-2}\left(1+\left(\frac{p}{3}\right)\right)-p^{2 k-3}+p^{2 k-4}\left(\left(\frac{p}{3}\right)+\left(\frac{p^{2}}{3}\right)\right)\right] a(1,1,1) } \\
& -p^{k-2} a\left(1, p, p^{2}\right)-p^{k-2} \sum_{\substack{\nu \bmod p \\
1+\nu+\nu^{2} \neq 0 \bmod p}} a\left(1+\nu+\nu^{2}, p(1+2 \nu), p^{2}\right) .
\end{aligned}
$$

The arithmetically interesting object associated with a Hecke eigenform is the Andrianov, or Spinor, zeta function:

Theorem [1, p. 165, Proposition 3.3.35; 2, Theorem 1.3.4, Theorem 2.2.1, and Corollary]. The space $M_{k}\left(\Gamma_{2}\right)$ has a basis consisting of simultaneous eigenforms for all Hecke operators $T(l)(l \in \mathbb{N})$. If $F$ is a simultaneous eigenform with eigenvalues $\lambda_{l}$, then the Andrianov zeta function

$$
Z_{F}(s):=\zeta(2 s-2 k+4) \sum_{l=1}^{\infty} \frac{\lambda_{l}}{l^{s}}
$$

has an Euler product of the form $Z_{F}(s)=\prod_{p} Q_{p}\left(p^{-s}\right)^{-1}$, where $Q_{p}(X)$ is a polynomial of degree 4:

$$
Q_{p}(X)=1-\lambda_{p} X+\left(\lambda_{p}^{2}-\lambda_{p^{2}}-p^{2 k-4}\right) X^{2}-\lambda_{p} p^{2 k-3} X^{3}+p^{4 k-6} X^{4} .
$$

Note that we can write the Euler factor $Q_{p}(X)$ in a more symmetric way as $Q_{p}(X)=\left(1-\left(\frac{\lambda_{p}}{2}+\sqrt{d_{p}}\right) X+p^{2 k-3} X^{2}\right)\left(1-\left(\frac{\lambda_{p}}{2}-\sqrt{d_{p}}\right) X+p^{2 k-3} X^{2}\right)$, 
where

$$
d_{p}=-\frac{3}{4} \lambda_{p}^{2}+\lambda_{p^{2}}+p^{2 k-4}+2 p^{2 k-3} .
$$

This Euler factor is said to fulfill the Ramanujan-Petersson conjecture if all its roots have absolute value $p^{\frac{3}{2}-k}$, i.e., if

$$
\left(\frac{\lambda_{p}}{2} \pm \sqrt{d_{p}}\right)^{2} \leq 4 p^{2 k-3}
$$

For the sake of completeness we mention the

Theorem [2, Theorem 2.4.1 and Theorem 3.1.1; 1, Theorem 4.3.16]. The Dirichlet series $Z_{F}(s)$ is absolutely convergent for $\Re(s) \gg 0$. It can be meromorphically continued to the complex plane and satisfies

$$
Z_{F}^{*}(s):=(2 \pi)^{-2 s} \Gamma(s) \Gamma(s-k+2) Z_{F}(s)=(-1)^{k} Z_{F}^{*}(2 k-2-s) .
$$

If $D<0$ is a fundamental discriminant, and $\chi$ a character of the group $K(D)$ of positive quadratic forms modulo $\mathrm{SL}_{2}(\mathbb{Z})$ with discriminant $D$, then

$$
\sum_{Q} \chi(Q) \sum_{l=1}^{\infty} \frac{a_{F}(l Q)}{l^{s}}=A_{\chi} L(s-k+2, \chi)^{-1} Z_{F}(s),
$$

where

$$
L(s, \chi)=\sum_{Q} \chi(Q) \sum_{l=1}^{\infty} \frac{r_{Q}(l)}{l^{s}}, \quad A_{\chi}=\sum_{Q} \chi(Q) a_{F}(Q)
$$

$\left(r_{Q}(l)=\right.$ number of representations of $l$ by $\left.Q\right)$. Here, the $Q$-sums are always over a complete set of representatives $Q$ for $K(D)$.

\section{HeCKe INVARIANT SPLITTINGS}

If we write an element $f$ of $M_{k}\left(\Gamma_{2}\right)$ in the form

$$
F=\sum_{m=0}^{\infty} \phi_{m} q^{\prime m}
$$

then the $\phi_{m}$ are known to be elements of $J_{k, m}$; the above expansion is the so-called Fourier-Jacobi expansion of $F$. The space of cusp forms $S_{k}\left(\Gamma_{2}\right)$ is the space of all such $F$ with $\phi_{0}=0$. This subspace is invariant under all Hecke operators. It contains the Hecke invariant subspace $V S_{k, 1}$. This subspace, in turn, is Hecke equivariantly isomorphic to $S_{2 k-2}\left(\Gamma_{1}\right)$ [5, $\left.\S 5\right]$.

By a result of Oda and Evdokimov, the subspace $V S_{k, 1}$ can be characterized as the subspace of $S_{k}\left(\Gamma_{2}\right)$ which is spanned by all those Hecke eigenforms whose $Z_{F}(s)$ has a pole (cf. [12]). From this it is clear that there exists one and only one Hecke invariant complement of $V S_{k, 1}$ in $S_{k}\left(\Gamma_{2}\right)$, namely the subspace spanned by all Hecke eigenforms $F$ with holomorphic $Z_{F}(s)$. We denote this space by $S_{k}^{?}\left(\Gamma_{2}\right)$.

Finally, for any elliptic cusp form $f$ in $S_{k}\left(\Gamma_{1}\right)$, one can form the KlingenEisenstein series

$$
K f=\left.\sum_{g \in \mathscr{C} \backslash \Gamma_{2}} \tilde{f}\right|_{k} g \quad\left(\tilde{f}\left(\tau, z, \tau^{\prime}\right):=f(\tau), \mathscr{C}=\mathscr{C}_{2,1}\right. \text { as in [8]). }
$$


The map $f \mapsto K f$ defines a Hecke equivariant embedding

$$
K: S_{k}\left(\Gamma_{1}\right) \hookrightarrow M_{k}\left(\Gamma_{2}\right) .
$$

It has the property that the 0th Fourier-Jacobi coefficient of $K f$ is $f$. In particular, we see that the dimension of $\mathbb{C} \cdot V E_{k} \oplus K S_{k}\left(\Gamma_{1}\right)$ equals the codimension of the subspace of cusp forms in $M_{k}\left(\Gamma_{2}\right)$, that this space contains no cusp forms, and hence, that this space is a Hecke invariant complement of the subspace of cusp forms.

Summarizing, one has the Hecke invariant splitting

$$
M_{k}\left(\Gamma_{2}\right)=K S_{k}\left(\Gamma_{1}\right) \oplus V J_{k, 1} \oplus S_{k}^{?}\left(\Gamma_{2}\right) .
$$

For the Andrianov zeta functions associated with the Hecke eigenforms in the former two spaces one knows the following (cf. $[8,5])$ :

$$
Z_{F}(s)= \begin{cases}L_{f}(s) L_{f}(s-k+2) & \text { for } F=K f, \\ L_{f}(s) L_{E_{2}}(s-k+2) & \text { for } F=V \phi,\end{cases}
$$

where, in the latter case, $f$ denotes a suitable Hecke eigenform in $S_{2 k-2}\left(\Gamma_{1}\right)$ and where, for any elliptic modular form $f$ (or $f=E_{2}$ ), we use

$$
L_{f}(s)=a_{f}(1)^{-1} \sum_{l=1}^{\infty} a_{f}(l) l^{-s}
$$

if $f=\sum_{l=0}^{\infty} a_{f}(l) q^{l}$ and $a_{f}(1) \neq 0$. The above identity shows in particular that the Ramanujan-Petersson conjecture fails for the Hecke eigenforms in $V S_{k, 1}$. The common expectation is that the Hecke eigenforms which satisfy the Ramanujan-Petersson conjecture are exactly those in the space $S_{k}^{?}\left(\Gamma_{2}\right)$.

Thus, it is clear that attention has to be focussed on the space of interesting Siegel modular forms $S_{k}^{?}\left(\Gamma_{2}\right)$.

\section{COMPUTATION OF THE FIRST INTERESTING HeCKe EIgENFORMS}

From Igusa's theorem we deduce that the dimension of $M_{k}\left(\Gamma_{2}\right)$ equals the number of quadruples $(a, b, c, d)$ of nonnegative integers satisfying $4 a+6 b+$ $10 c+12 d=k$, i.e.,

$$
\sum_{k=0}^{\infty} \operatorname{dim} M_{2 k}\left(\Gamma_{2}\right) X^{2 k}=\frac{1}{\left(1-X^{4}\right)\left(1-X^{6}\right)\left(1-X^{10}\right)\left(1-X^{12}\right)}
$$

Similarily, using

$$
S_{*}\left(\Gamma_{1}\right):=\bigoplus_{k \in \mathbb{Z}} S_{k}\left(\Gamma_{1}\right)=\Delta \cdot \mathbb{C}\left[E_{4}, E_{6}\right]
$$

and the fact that $K, V$ are injective and that $S_{2 k, 1}$ is isomorphic to $S_{4 k-2}\left(\Gamma_{1}\right)$, we find

$$
\begin{aligned}
\sum_{k=0}^{\infty} \operatorname{dim} K S_{2 k}\left(\Gamma_{1}\right) X^{2 k} & =\frac{X^{12}}{\left(1-X^{4}\right)\left(1-X^{6}\right)} \\
\sum_{k=0}^{\infty} \operatorname{dim} V S_{2 k, 1} X^{2 k} & =\frac{X^{10}\left(1+X^{2}\right)}{\left(1-X^{4}\right)\left(1-X^{6}\right)}
\end{aligned}
$$


and finally, by the results quoted in the preceding section,

$$
\sum_{k=0}^{\infty} \operatorname{dim} S_{2 k}^{?}\left(\Gamma_{2}\right) X^{2 k}=\frac{X^{20}\left(1+X^{2}+X^{4}-X^{12}-X^{14}\right)}{\left(1-X^{4}\right)\left(1-X^{6}\right)\left(1-X^{10}\right)\left(1-X^{12}\right)}
$$

Table 1 in $\S 6$ lists the dimensions of the first few Hecke invariant subspaces.

The first candidate for an interesting Hecke eigenform, i.e., a non-MaaßSpezialschar cusp eigenform, is found in weight 20 . Since $S_{20}^{?}\left(\Gamma_{2}\right)$ is onedimensional, this first non-Spezialschar cusp eigenform is uniquely determined (up to multiplication by scalars)-we call it $\Upsilon 20$. In [10] its first few Hecke eigenvalues have been computed (our $\Upsilon 20$ equals $-\frac{1}{2}$ times Kurokawa's $\chi_{20}^{(3)}$ ). To write down a formula for it, we note first of all that the cusp form $\chi_{10}^{2}$ is not a Maaß-Spezialscharform: in fact, its Fourier-Jacobi expansion starts with $\phi_{10}^{2} q^{\prime 2}+\cdots$, i.e., its first Fourier-Jacobi coefficient vanishes, whereas the first Fourier-Jacobi coefficient of a Maaß-Spezialform $V \phi$ is $\phi$ itself.

Thus, $\chi_{10}^{2}$ equals $\Upsilon 20$ plus a Maaß-Spezialscharform, i.e., $\Upsilon 20$ can be obtained by adding a suitable cusp Maaß-Spezialscharform to $\chi_{10}^{2}$. The subspace $V S_{20,1}$ of Spezialscharformen in $S_{20}\left(\Gamma_{2}\right)$ is two-dimensional; it is spanned by $V\left(\phi_{10} E_{4} E_{6}\right)$ and $V\left(\phi_{12} E_{4}^{2}\right)$. Hence, up to normalization,

$$
\Upsilon 20 \approx \chi_{10}^{2}+a V\left(\phi_{10} E_{4} E_{6}\right)+b V\left(\phi_{12} E_{4}^{2}\right)
$$

for suitable constants $a$ and $b$. To find $a$ and $b$, we computed sufficiently many coefficients of $\chi_{10}^{2}, V\left(\phi_{10} E_{4} E_{6}\right)$, and $V\left(\phi_{12} E_{4}^{2}\right)$. Then we applied $T(2)$ to these forms, using the formula for $T(p)$ in $\S 2$. This enabled us to find the matrix $M$, which is uniquely determined by $T(2) B=M B$, where $B=\left(\chi_{10}^{2}, V\left(\phi_{10} E_{4} E_{6}\right), V\left(\phi_{12} E_{4}^{2}\right)\right)^{t}$. By well-known algebra we have $\chi(M) B=$ $v \cdot \Upsilon 20$ with a suitable complex column vector $v$, where $\chi(X)$ is the characteristic polynomial of the restriction of $T(2)$ to $V S_{20,1}$. From the formula for the Andrianov zeta function of a Maßß-Spezialform quoted in $\S 3$ one verifies for the latter polynomial the identity $\chi(X)=\tilde{\chi}\left(X+2^{k-2}+2^{k-1}\right)$, where $\tilde{\chi}(X)$ denotes the characteristic polynomial of the Hecke operator $T(2)$ on the space $S_{38}\left(\Gamma_{1}\right)$ of elliptic cusp forms of weight 38 . The latter can be computed by well-known procedures.

The other first few Hecke eigenforms $\Upsilon 22, \Upsilon 24 a, \Upsilon 24 b, \Upsilon 26 a, \Upsilon 26 b, \ldots$ of weights 22 to 32 can be found similarly. The particular results are given in Table 2. In Table 3 we list the first few Fourier coefficients of these forms.

Note that Table 2 shows in particular that all the forms $\Upsilon 22$ up to $\Upsilon 26 b$ have rational Fourier coefficients. For the Hecke eigenforms in $S_{28}^{?}\left(\Gamma_{2}\right), S_{30}^{?}\left(\Gamma_{2}\right)$, $S_{32}^{?}\left(\Gamma_{2}\right)$, this is not true; their Fourier coefficients generate (after suitable normalization) a cubic, quartic, quintic number field, respectively. This is easily deduced from the fact that the characteristic polynomials $H k(X)$ of $T(2)$ on $S_{k}^{?}\left(\Gamma_{2}\right)(k=28,30,32)$ are irreducible over $\mathbb{Q}$. These characteristic polynomials are listed in Table 5 . This table also gives the prime decomposition of the discriminants $f d k$ of the fields $\mathbb{Q}[X] /(H k(X))$. Note, that these discriminants contain only a small number of primes as compared to their impressive size. It may be worthwhile to investigate whether this is part of a more general phenomenon. 
Finally, using the formulas for $\lambda_{p}$ and $\lambda_{p^{2}}$ from $\S 2$, one can compute the first few Euler factors $Q_{p}(X)$ of the Andrianov zeta function of $\Upsilon 22$ up to $\Upsilon 26 b$. The resulting values of $\lambda_{p}$ and $d_{p}$ are given in Table 4 .

We checked within the range of Table 4 that the roots of $X^{2}-\left(\frac{\lambda_{p}}{2} \pm \sqrt{d_{p}}\right) X+$ $p^{2 k-3}$ are complex conjugate. Thus all roots of $Q_{p}(X)$ have absolute value $p^{\frac{3}{2}-k}$, i.e., within the range of our computations, the eigenforms $\Upsilon 20$ to $\Upsilon 26 b$ satisfy the generalized Ramanujan-Petersson conjecture.

\section{CONGRUenCES FOR THE INTERESTING HeCKe eIGENFORMS}

A Siegel modular form is said to be defined over $R$ (a subring of $\mathbb{C}$ ) if all its Fourier coefficients are contained in $R$, i.e., if its Fourier expansion can be viewed as an element of $R \llbracket q, \zeta, q^{\prime} \rrbracket$. Two Siegel modular forms which are defined over $\mathbb{Z}$ are said to be congruent modulo $N(\in \mathbb{Z})$ if they have the same image under the projection map

$$
\mathbb{Z} \llbracket q, \zeta, q^{\prime} \rrbracket \rightarrow \mathbb{Z} \llbracket q, \zeta, q^{\prime} \rrbracket / N \mathbb{Z} \llbracket q, \zeta, q^{\prime} \rrbracket .
$$

A similar obvious terminology will be applied to Jacobi forms, elliptic modular forms, and Dirichlet series.

Using this terminology, we note the following

Proposition. All the Siegel modular forms $\Upsilon 20-\Upsilon 26 b$ listed in Table 2 are defined over $\mathbb{Z}$. One has

$$
a_{\Upsilon *}(1,1,1)= \begin{cases}1 & \text { if } *=20,22,24 a, 26 a, \\ 3 & \text { if } *=24 b, 26 b .\end{cases}
$$

For each of these forms the g.c.d. of its Fourier coefficients is 1.

Proof. These assertions are easily read off from Tables 2 and 3. For the first assertion one uses the following obvious facts: The Jacobi forms $\phi_{10}$ and $\phi_{12}$ and the elliptic modular forms occurring in Table 2 are defined over $\mathbb{Z}$. The $V$-operator maps forms defined over $\mathbb{Z}$ to forms defined over $\mathbb{Z}$. Therefore, all Maaß-Spezialscharformen occurring in Table 2 are defined over $\mathbb{Z}$. Thus the $\Upsilon_{*}$ are $\frac{1}{6} \mathbb{Z}$-linear combinations of forms defined over $\mathbb{Z}$, i.e., they have rational Fourier coefficients with denominators at most equal to 6 . That the denominator 6 does not really occur has to be checked case by case, using the fact that $E_{4}$ and $E_{6}$ are congruent to 1 modulo 24 , and $\phi_{10}$ and $\phi_{12}$ are congruent modulo 12 . The latter is immediately clear from the formulas in Notation.

This proposition, together with Table 2 , immediately implies that $\Upsilon 20$ is congruent modulo $2^{9} \cdot 3^{2} \cdot 5 \cdot 7 \cdot 11$ to the Spezialscharform $V\left(\frac{1}{2} \phi_{12} E_{4}^{2}+\frac{1}{2} \phi_{10} E_{4} E_{6}\right)$, that $\Upsilon 22$ is congruent to a Spezialscharform modulo $2^{5} \cdot 3 \cdot 5 \cdot 7 \cdot 1423$, etc. Even more, it is clear from the explicit formulas in Table 2 that the number $2^{9} \cdot 3^{2} \cdot 5 \cdot 7 \cdot 11$ is divisible by any $N$ such that $\Upsilon 20$ is congruent modulo $N$ to a Spezialscharform, and similar statements also hold for the other eigenforms in Table 2.

It is not hard to prove that congruences such as the ones just considered imply congruences for the Andrianov zeta functions. More precisely, one has 
Proposition. Let $N$ be a positive integer, and let $F \in V J_{k, 1}$ be defined over $\mathbb{Z}$ and such that the g.c.d. of its Fourier coefficients is prime to $N$. Assume that $F$ is a Hecke eigenform modulo $N$, i.e., that $T(l) F \equiv \lambda_{l} F \bmod N$ for all $l$ and with suitable integers $\lambda_{l}$. Then there exists an $f \in S_{2 k-2}\left(\Gamma_{1}\right)$, which is defined over $\mathbb{Z}$ and is a Hecke eigenform modulo $N$, such that

$$
\zeta(2 s-2 k+4) \sum_{l=1}^{\infty} \frac{\lambda_{l}}{l s} \equiv L_{f}(s) L_{E_{2}}(s-k+2) \quad \bmod N .
$$

Proof. If $F$ is a Hecke eigenform, one has for any $Q$

$$
\zeta(2 s-2 k+4) \sum_{l=1}^{\infty} a_{T(l) F}(Q) l^{-s}=a_{F}(Q) Z_{F}(s),
$$

whence

$$
\zeta(2 s-2 k+4) \sum_{l=1}^{\infty} a_{T(l) F}(Q) l^{-s}=a_{F}(Q) L_{f}(s) L_{E_{2}}(s-k+2)
$$

with a suitable elliptic modular form $f$ from $M_{2 k-2}\left(\Gamma_{1}\right)$. Since $V J_{k, 1}$ has a basis of Hecke eigenforms, and by linearity, the latter identity is true for any element in $V J_{k, 1}$. In particular, it holds true for the $F$ as in the proposition, and since $a_{T(l) F}(Q) \equiv \lambda_{l} a_{F}(Q) \bmod N$, we conclude

$$
\zeta(2 s-2 k+4) a_{F}(Q) \sum_{l=1}^{\infty} \lambda_{l} l^{-s} \equiv a_{F}(Q) L_{f}(s) L_{E_{2}}(s-k+2) \quad \bmod N .
$$

Note that by assumption on $F$, and the foregoing identity, $f$ is defined over $\mathbb{Z}$. Since by assumption the g.c.d. of the $a_{F}(Q)$ and $N$ are relatively prime, we deduce from the last identity the asserted one. This identity shows in particular that $L_{f}(s) \bmod N$ has an Euler product, and by well-known arguments this implies that $f$ is a Hecke eigenform modulo $N$.

As we saw above, the $\Upsilon *$ are congruent to Spezialscharformen modulo certain $N$. These Spezialscharformen are then Hecke eigenforms modulo $N$, and their Fourier coefficients are even relatively prime (cf. Table 3), i.e., they fulfill exactly the assumptions of the proposition. Thus, the proposition shows that to each $\Upsilon_{*}$ and its associated $N$, there corresponds an elliptic modular form $f$, which is a Hecke eigenform modulo $N$, such that

$$
Z_{\Upsilon *}(s) \equiv L_{f}(s) L_{E_{2}}(s-k+2) \quad \bmod N .
$$

Note that this identity implies $a_{f}(p) \equiv \lambda_{p}-p^{k-2}-p^{k-1} \bmod N$, where $p$ denotes any prime and $\lambda_{p}$ the eigenvalue of $\Upsilon k *$ with respect to $T(p)$. Thus, given $N$ and the first few eigenvalues $\lambda_{p}$ of $\Upsilon k *$, we can immediately identify the modular form $f$ mod $N$ with respect to any $\mathbb{Z}$-basis of the lattice of elements of $S_{2 k-2}\left(\Gamma_{1}\right)$ which are defined over $\mathbb{Z}$. The particular $f$ (and $N$ ) corresponding to the $\Upsilon_{*}$ are listed in Table 6 . 
Note that these congruences, together with the theory of congruences for elliptic modular forms, imply further congruences. For example, from the first row of Table 6 we can deduce the congruences

$$
\begin{array}{ll}
Z_{\Upsilon 20}(s) \equiv L_{E_{2}}(s-18) L_{E_{2}}(s-4) & \bmod 5, \\
Z_{\Upsilon 20}(s) \equiv L_{E_{2}}(s-18) L_{E_{2}}(s-3) & \bmod 7 \\
Z_{\Upsilon 20}(s) \equiv L_{E_{2}}(s-18) L_{E_{4}}(s-2) & \bmod 11 .
\end{array}
$$

To prove these congruences, recall first of all that for any prime $p$ one has $E_{2} \equiv E_{p+1} \bmod p$, and that $\theta:=q \frac{d}{d q} \operatorname{maps} \widetilde{M}_{k}\left(\Gamma_{1}\right)$ to $\widetilde{S}_{k+p+1}\left(\Gamma_{1}\right)$, preserving Hecke eigenforms. Here, $\widetilde{M}_{k}\left(\Gamma_{1}\right)$ and $\widetilde{S}_{k+p+1}\left(\Gamma_{1}\right)$ denote the reduction modulo $p$ of the $\mathbb{Z}$-modules of modular forms in $M_{k}\left(\Gamma_{1}\right)$ and $S_{k+p+1}\left(\Gamma_{1}\right)$, respectively, which have Fourier coefficients in $\mathbb{Z}$. From this it is immediately clear that $\theta^{2} E_{2} \bmod 5, \theta^{4} E_{2} \bmod 5$, or $\theta^{3} E_{2} \bmod 7, \theta^{2} E_{4} \bmod 7$, or $\theta^{2} E_{4} \bmod 11, \theta E_{6} \bmod 11$ are Hecke eigenforms in $\widetilde{S}_{38}\left(\Gamma_{1}\right)$ for $p=5,7,11$, respectively. Since the latter spaces are two-dimensional (over $\mathbb{Z} / p \mathbb{Z}$ ), these are all Hecke eigenforms in these spaces, and hence the $f$ in Table 6 has to be congruent modulo $5,7,11$ to one of these eigenforms (up to multiplication by a scalar), respectively. The particular congruences, which one finds in each of these cases, are just the ones listed above.

We leave it to the reader to verify similar congruences for the other $\Upsilon *$.

Finally, we mention another kind of congruence which can immediately be read off from Table 2 . Namely, if we look at the $2 \times 5$ matrix which has as rows the rows of Table 2 corresponding to $\Upsilon 24 a$ and $\Upsilon 24 b$, then we recognize that the g.c.d. of its $2 \times 2$ minors is $4 \cdot 31$. This indicates that there should be a congruence between $\Upsilon 24 a$ and $\Upsilon 24 b$ modulo $4 \cdot 31$, and that $4 \cdot 31$ is the largest integer for which such a congruence holds true. In fact, consulting Table 2 , one easily verifies the congruence

$$
3 \cdot \Upsilon 24 a \equiv \Upsilon 24 b \quad \bmod 4 \cdot 31 ;
$$

indeed, the coefficients $3 \cdot\left(-2^{5} \cdot 3^{2} \cdot 5 \cdot 7 \cdot 11 \cdot 157\right)$ and $-2^{7} \cdot 3 \cdot 7 \cdot 13^{2} \cdot 83$ in the formulas for $3 \cdot \Upsilon 24 a$ and $\Upsilon 24 b$ in front of $\chi_{10} \chi_{14}$ are congruent modulo 31 , and the same is true for the corresponding coefficients in front of $\chi_{12}^{2}$, $\phi_{12} E_{6}^{2}$, etc. The claimed congruence modulo 4 can be verified similarily by using additionally that $\phi_{12} E_{6}^{2}$ is congruent modulo 4 to $\phi_{10} E_{4}^{2} E_{6}$. In the same way, it is deduced that

$$
3 \cdot \Upsilon 26 a \equiv \Upsilon 26 b \bmod 4 \cdot 37
$$

and that $4 \cdot 37$ is the largest integer for which such a congruence holds true. It is easily checked (e.g., by using the formula expressing the Spinor zeta function in terms of Fourier coefficients, as quoted in the last theorem in $\S 2$ ) that these congruences imply corresponding congruences for the Spinor zeta functions. 
6. TABLES

TABLE 1

Dimensions of $M_{k}\left(\Gamma_{2}\right)$ and subspaces for $0 \leq k \leq 50$

\begin{tabular}{|c|c|c|c|c|}
\hline$k$ & $M_{k}\left(\Gamma_{2}\right)$ & $K S_{k}\left(\Gamma_{1}\right)$ & $V S_{k, 1}$ & $S_{k}^{?}\left(\Gamma_{2}\right)$ \\
\hline 0 & 1 & - & - & - \\
\hline 2 & - & - & - & - \\
\hline 4 & 1 & - & - & - \\
\hline 6 & 1 & - & - & - \\
\hline 8 & 1 & - & - & - \\
\hline 10 & 2 & - & 1 & - \\
\hline 12 & 3 & 1 & 1 & - \\
\hline 14 & 2 & 0 & 1 & - \\
\hline 16 & 4 & 1 & 2 & - \\
\hline 18 & 4 & 1 & 2 & - \\
\hline 20 & 5 & 1 & 2 & 1 \\
\hline 22 & 6 & 1 & 3 & 1 \\
\hline 24 & 8 & 2 & 3 & 2 \\
\hline 26 & 7 & 1 & 3 & 2 \\
\hline 28 & 10 & 2 & 4 & 3 \\
\hline 30 & 11 & 2 & 4 & 4 \\
\hline 32 & 12 & 2 & 4 & 5 \\
\hline 34 & 14 & 2 & 5 & 6 \\
\hline 36 & 17 & 3 & 5 & 8 \\
\hline 38 & 16 & 2 & 5 & 8 \\
\hline 40 & 21 & 3 & 6 & 11 \\
\hline 42 & 22 & 3 & 6 & 12 \\
\hline 44 & 24 & 3 & 6 & 14 \\
\hline 46 & 27 & 3 & 7 & 16 \\
\hline 48 & 31 & 4 & 7 & 19 \\
\hline 50 & 31 & 3 & 7 & 20 \\
\hline
\end{tabular}


TABLE 2

Explicit formulas for the interesting Hecke eigenforms $\Upsilon 20-\Upsilon 26 b$

$$
\begin{aligned}
\Upsilon 20= & -2^{9} \cdot 3^{2} \cdot 5 \cdot 7 \cdot 11 \cdot \chi_{10}^{2}+V\left(\frac{1}{2} \phi_{12} E_{4}^{2}+\frac{1}{2} \phi_{10} E_{4} E_{6}\right), \\
\Upsilon 22= & -2^{5} \cdot 3 \cdot 5 \cdot 1423 \cdot \chi_{10} \chi_{12} \\
& +V\left(-\frac{5}{2 \cdot 3} \phi_{12} E_{4} E_{6}+\frac{11}{2 \cdot 3} \phi_{10} E_{6}^{2}+2^{4} \cdot 3 \cdot 61 \cdot \phi_{10} \Delta\right) \\
\Upsilon 24 a= & -2^{5} \cdot 3^{2} \cdot 5 \cdot 7 \cdot 11 \cdot 157 \cdot \chi_{10} \chi_{14}+2^{5} \cdot 3 \cdot 5^{2} \cdot 11 \cdot 157 \cdot \chi_{12}^{2} \\
& +V\left(-\frac{7}{2 \cdot 3} \phi_{12} E_{6}^{2}-2^{4} \cdot 3 \cdot 67 \cdot \phi_{12} \Delta+\frac{13}{2 \cdot 3} \phi_{10} E_{4}^{2} E_{6}\right), \\
\Upsilon 24 b= & -2^{7} \cdot 3 \cdot 7 \cdot 13^{2} \cdot 83 \cdot \chi_{10} \chi_{14}-2^{6} \cdot 3 \cdot 7 \cdot 13^{2} \cdot 83 \cdot \chi_{12}^{2} \\
& +V\left(\frac{41}{2 \cdot 3} \cdot \phi_{12} E_{6}^{2}+2^{6} \cdot 3 \cdot 5 \cdot 7 \cdot \phi_{12} \Delta-\frac{23}{2 \cdot 3} \phi_{10} E_{4}^{2} E_{6}\right), \\
\Upsilon 26 a= & -2^{6} \cdot 3^{3} \cdot 5^{2} \cdot 11 \cdot 29 \cdot \chi_{10} \chi_{16 a}-2^{6} \cdot 3^{4} \cdot 5^{2} \cdot 11 \cdot 29 \cdot \chi_{12} \chi_{14} \\
& +V\left(-\frac{1}{2} \phi_{12} E_{4}^{2} E_{6}+\frac{3}{2} \phi_{10} E_{4}^{4}-2^{5} \cdot 3^{2} \cdot 31 \cdot \phi_{10} \Delta E_{4}\right), \\
\Upsilon 26 b= & -2^{6} \cdot 3^{3} \cdot 5^{3} \cdot 7 \cdot 13^{2} \cdot \chi_{10} \chi_{16 a}+2^{5} \cdot 3^{3} \cdot 5^{2} \cdot 7^{2} \cdot 13^{2} \cdot \chi_{12} \chi_{14} \\
& +V\left(\frac{5 \cdot 13}{2 \cdot 3} \cdot \phi_{12} E_{4}^{2} E_{6}-\frac{47}{2 \cdot 3} \phi_{10} E_{4}^{4}+2^{4} \cdot 3 \cdot 5 \cdot 251 \cdot \phi_{10} \Delta E_{4}\right)
\end{aligned}
$$

TABLE 3

The first few Fourier coefficients of $\Upsilon 20-\Upsilon 26 b$

\begin{tabular}{l|rrrrr}
$\Upsilon *$ & $1,1,1$ & $1,0,1$ & \multicolumn{1}{c}{$1,1,2$} & \multicolumn{1}{c}{$1,0,2$} & \multicolumn{1}{c}{$1,1,3$} \\
\hline$\Upsilon 20$ & 1 & $2^{2}$ & $2^{3} \cdot 7$ & $2^{3} \cdot 3 \cdot 109$ & $-3 \cdot 11 \cdot 1669$ \\
$\Upsilon 22$ & 1 & $-2^{2} \cdot 3$ & $2^{6} \cdot 3 \cdot 7$ & $2^{3} \cdot 3^{3}$ & $3^{4} \cdot 5059$ \\
$\Upsilon 24 a$ & 1 & $-2^{4}$ & $-2^{3} \cdot 11 \cdot 23$ & $-2^{5} \cdot 3 \cdot 11 \cdot 19$ & $-3 \cdot 11 \cdot 23 \cdot 563$ \\
$\Upsilon 24 b$ & 3 & $2^{2} \cdot 19$ & $-2^{3} \cdot 7 \cdot 11$ & $-2^{3} \cdot 3 \cdot 11^{2}$ & $3 \cdot 11 \cdot 131 \cdot 491$ \\
$\Upsilon 26 a$ & 1 & $-2^{3}$ & $-2^{5} \cdot 233$ & $2^{4} \cdot 3 \cdot 317$ & $-3 \cdot 11 \cdot 83 \cdot 431$ \\
$\Upsilon 26 b$ & 3 & $2^{2} \cdot 31$ & $2^{4} \cdot 7 \cdot 461$ & $-2^{3} \cdot 3 \cdot 17 \cdot 269$ & $3 \cdot 2433059$
\end{tabular}




\section{TABLE 4}

The first few Euler factors of the Andrianov zeta functions of $\Upsilon 20-\Upsilon 26 b$

$$
\begin{aligned}
Z_{\Upsilon *}(s)=\prod_{p} & \left(1-\left(\frac{\lambda_{p}}{2}+\sqrt{d_{p}}\right) p^{-s}+p^{2 k-3-2 s}\right)^{-1} \\
& \cdot\left(1-\left(\frac{\lambda_{p}}{2}-\sqrt{d_{p}}\right) p^{-s}+p^{2 k-3-2 s}\right)^{-1}
\end{aligned}
$$

\begin{tabular}{|c|c|c|c|}
\hline$\Upsilon *$ & $p$ & $\lambda_{p}$ & $d_{p}=-\frac{3}{4} \lambda_{p}^{2}+\lambda_{p^{2}}+p^{2 k-4}+2 p^{2 k-3}$ \\
\hline \multirow[t]{4}{*}{$\Upsilon 20$} & 2 & $-2^{8} \cdot 3^{2} \cdot 5 \cdot 73$ & $2^{14} \cdot 3^{2} \cdot 7 \cdot 13 \cdot 19 \cdot 241$ \\
\hline & 3 & $2^{3} \cdot 3^{5} \cdot 5 \cdot 7 \cdot 5099$ & $2^{6} \cdot 3^{10} \cdot 19 \cdot 47 \cdot 150628997$ \\
\hline & 5 & $-2^{2} \cdot 3^{2} \cdot 5^{3} \cdot 7 \cdot 166103087$ & $2^{8} \cdot 3^{2} \cdot 5^{6} \cdot 19 \cdot 47 \cdot 1396135808326877$ \\
\hline & 7 & $2^{4} \cdot 5^{2} \cdot 7^{3} \cdot 673 \cdot 28346749$ & $2^{8} \cdot 3^{6} \cdot 7^{6} \cdot 29 \cdot 1097 \cdot 41713094306662453$ \\
\hline \multirow[t]{3}{*}{$\Upsilon 22$} & 2 & $-2^{8} \cdot 3 \cdot 5 \cdot 577$ & $2^{14} \cdot 3^{4} \cdot 13^{2} \cdot 31 \cdot 439$ \\
\hline & 3 & $-2^{3} \cdot 3^{5} \cdot 5 \cdot 19 \cdot 97 \cdot 167$ & $2^{8} \cdot 3^{10} \cdot 11 \cdot 61 \cdot 8364437759$ \\
\hline & 5 & $2^{2} \cdot 3 \cdot 5^{3} \cdot 60700091989$ & $2^{10} \cdot 3^{4} \cdot 5^{6} \cdot 7193 \cdot 9888524030928593$ \\
\hline \multirow[t]{4}{*}{$\Upsilon 24 a$} & 2 & $-2^{11} \cdot 3 \cdot 5 \cdot 181$ & $2^{20} \cdot 3^{2} \cdot 7 \cdot 17 \cdot 61559$ \\
\hline & 3 & $-2^{3} \cdot 3^{6} \cdot 5 \cdot 7 \cdot 23^{2} \cdot 491$ & $2^{6} \cdot 3^{12} \cdot 7^{3} \cdot 413057028823$ \\
\hline & 5 & $-2^{2} \cdot 3 \cdot 5^{3} \cdot 7 \cdot 29 \cdot 109438961$ & $2^{8} \cdot 3^{2} \cdot 5^{6} \cdot 7^{2} \cdot 13 \cdot 19^{2} \cdot 157 \cdot 659$ \\
\hline & & & .74293331977811 \\
\hline \multirow[t]{3}{*}{$\Upsilon 24 b$} & 2 & $-2^{9} \cdot 3^{2} \cdot 23 \cdot 61$ & $2^{16} \cdot 3^{2} \cdot 5 \cdot 11^{2} \cdot 97 \cdot 373$ \\
\hline & 3 & $-2^{3} \cdot 3^{6} \cdot 2328401$ & $2^{6} \cdot 3^{12} \cdot 5 \cdot 11^{2} \cdot 13^{2} \cdot 1163672669$ \\
\hline & 5 & $2^{2} \cdot 3^{2} \cdot 5^{3} \cdot 1562781531383$ & $2^{8} \cdot 3^{2} \cdot 5^{6} \cdot 11^{2} \cdot 13^{2} \cdot 50368985463609956441$ \\
\hline \multirow[t]{4}{*}{$\Upsilon 26 a$} & 2 & $-2^{13} \cdot 3^{2} \cdot 5 \cdot 7^{2}$ & $2^{24} \cdot 3^{2} \cdot 859 \cdot 5779$ \\
\hline & 3 & $-2^{3} \cdot 3^{5} \cdot 5 \cdot 307 \cdot 61091$ & $2^{10} \cdot 3^{10} \cdot 107 \cdot 1093 \cdot 16123577711$ \\
\hline & 5 & $-2^{2} \cdot 3^{2} \cdot 5^{5} \cdot 13 \cdot 37 \cdot 293$ & $2^{12} \cdot 3^{2} \cdot 5^{10} \cdot 17 \cdot 373 \cdot 165515489$ \\
\hline & & $\cdot 1847 \cdot 3067$ & $\cdot 74684067301$ \\
\hline \multirow[t]{4}{*}{$\Upsilon 26 b$} & 2 & $-2^{9} \cdot 3^{2} \cdot 5 \cdot 229$ & $2^{16} \cdot 3^{2} \cdot 7 \cdot 67 \cdot 163 \cdot 33703$ \\
\hline & 3 & $-2^{3} \cdot 3^{7} \cdot 5 \cdot 7 \cdot 1061 \cdot 1579$ & $2^{10} \cdot 3^{14} \cdot 41 \cdot 1153 \cdot 594719897$ \\
\hline & 5 & $2^{2} \cdot 3^{2} \cdot 5^{3} \cdot 7 \cdot 37 \cdot 757$ & $2^{12} \cdot 3^{2} \cdot 5^{6} \cdot 11 \cdot 206009$ \\
\hline & & $\cdot 2713 \cdot 51713$ & $\cdot 13183364794216242331$ \\
\hline
\end{tabular}




\section{TABLE 5}

Characteristic polynomials $H k$ of $T(2)$ on $S_{k}^{?}\left(\Gamma_{2}\right)$ and discriminants fdk of $\mathbb{Q}[X] /(H k(X))$ for $k=28,30,32$

$$
\begin{aligned}
H 28= & X^{3}+137681664 X^{2}+4794374687293440 X \\
& +4100431555335920025600 \\
f d 28= & 5 \cdot 13 \cdot 73693 \cdot 1418741 \\
H 30= & X^{4}+374036736 X^{3}-38240213642772480 X^{2} \\
& -1675860454758443227545600 X \\
& +3326494782878021681883906048000 \\
f d 30= & 3 \cdot 769896956241058733183 \\
H 32= & X^{5}+2026982400 X^{4}-1037849863848984576 X^{3} \\
& -1460765778655696250606714880 X^{2} \\
& +197850685506224024897745617682432000 X \\
& +186323642358004277344714415914598409437184000 \\
f d 32= & 2^{2} \cdot 3 \cdot 7 \cdot 170912892945636421076635084794644759
\end{aligned}
$$

TABLE 6

Congruences for the Andrianov zeta functions $Z_{\Upsilon_{*}}(s) \equiv$ $L_{E_{2}}(s-k+2) L_{f}(s) \bmod N$

\begin{tabular}{l|l|r}
\hline$\Upsilon *$ & \multicolumn{1}{|c|}{$f$} & \multicolumn{1}{|c}{$N$} \\
\hline$\Upsilon 20$ & $E_{4}^{5} \cdot E_{6} \cdot \Delta+146016 E_{4}^{2} \cdot E_{6} \cdot \Delta^{2}$ & $2^{9} \cdot 3^{2} \cdot 5 \cdot 7 \cdot 11$ \\
$\Upsilon 22$ & $E_{4}^{6} \cdot E_{6} \cdot \Delta+4200240 E_{4}^{3} \cdot E_{6} \cdot \Delta^{2}+4200240 E_{6} \cdot \Delta^{3}$ & $2^{5} \cdot 3 \cdot 5 \cdot 7 \cdot 1423$ \\
$\Upsilon 24 a$ & $E_{4}^{7} \cdot E_{6} \cdot \Delta+92736 E_{4}^{4} \cdot E_{6} \cdot \Delta^{2}+33120 E_{4} \cdot E_{6} \cdot \Delta^{3}$ & $2^{5} \cdot 3 \cdot 5 \cdot 11 \cdot 157$ \\
$\Upsilon 24 b$ & $E_{4}^{7} \cdot E_{6} \cdot \Delta+18655488 E_{4}^{4} \cdot E_{6} \cdot \Delta^{2}+12111936 E_{4} \cdot E_{6} \cdot \Delta^{3}$ & $2^{6} \cdot 3 \cdot 7 \cdot 13^{2} \cdot 83$ \\
$\Upsilon 26 a$ & $E_{4}^{8} \cdot E_{6} \cdot \Delta+507600 E_{4}^{5} \cdot E_{6} \cdot \Delta^{2}+13694400 E_{4}^{2} \cdot E_{6} \cdot \Delta^{3}$ & $2^{6} \cdot 3^{3} \cdot 5^{2} \cdot 11 \cdot 29$ \\
$\Upsilon 26 b$ & $E_{4}^{8} \cdot E_{6} \cdot \Delta+46602000 E_{4}^{5} \cdot E_{6} \cdot \Delta^{2}+22420800 E_{4}^{2} \cdot E_{6} \cdot \Delta^{3}$ & $2^{6} \cdot 3^{3} \cdot 5^{2} \cdot 7 \cdot 13^{2}$ \\
\hline
\end{tabular}




\section{BIBLIOGRAPHY}

1. A. N. Andrianov, Quadratic forms and Hecke operators, Springer, Berlin, 1987.

2. __ Euler products corresponding to Siegel modular forms of genus 2, Russian Math. Surveys 29 (1974), no. 3, 45-116.

3. C. Batut, D. Bernardi, H. Cohen, and M. Olivier, PARI-GP, Université Bordeaux 1, Bordeaux, 1989.

4. H. Cohen, N.-P. Skoruppa, and D. Zagier, Tables of modular forms, Springer-Verlag, 1992 (to appear).

5. M. Eichler and D. Zagier, The theory of Jacobi forms, Birkhäuser, Boston, 1985.

6. E. Freitag, Siegelsche Modulfunktionen, Springer, Berlin, 1983.

7. J.-I. Igusa, On Siegel modular forms of genus two, Amer. J. Math. 84 (1962), 175-200.

8. H. Klingen, Zum Darstellungssatz für Siegelsche Modulformen, Math. Z. 102 (1967), 20-43; Berichtigung, Math. Z. 105 (1967), 399-400.

9. W. Kohnen and N.-P. Skoruppa, A certain Dirichlet series attached to Siegel modular forms of degree two, Invent. Math. 95 (1989), 541-558.

10. N. Kurokawa, Examples of eigenvalues of Hecke operators on Siegel cusp forms of degree two, Invent. Math. 49 (1978), 149-165.

11. H. Maaß, Über eine Spezialschar von Modulformen zweiten Grades. I, II, III, Invent. Math. 52 (1979), 95-104; 53 (1979), 249-253, 255-265.

12. T. Oda, On the poles of Andrianov L-functions, Math. Ann. 256 (1981), 323-340.

13. N.-P. Skoruppa, Über den Zusammenhang zwischen Jacobiformen und Modulformen halbganzen Gewichts, Bonner Math. Schriften, no. 159, Univ. Bonn, Bonn, 1985.

Max-Planck-Institut für Mathematik, Gottfried-Claren-Strasse 26, D-5300 Bonn 3, GERMANY

Mathematisches Institut der Universität, Wegelerstrasse 10, D-5300 BonN 1, GerMANY

E-mail address: nils@mpim-bonn.mpg.dbp.de 